\title{
Article \\ Effects of Deoxynivalenol and Fumonisins Fed in Combination
to Beef Cattle: Immunotoxicity and Gene Expression
}

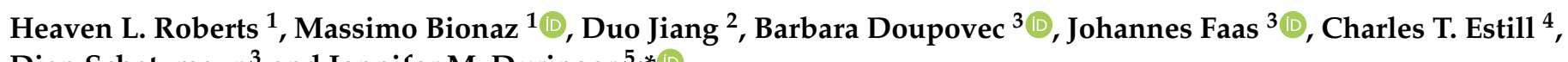
Dian Schatzmayr ${ }^{3}$ and Jennifer M. Duringer ${ }^{5, *} * \mathbb{D}$

1 Department of Animal \& Rangeland Sciences, College of Agricultural Sciences, Oregon State University, Corvallis, OR 97331, USA; heavenlearoberts@gmail.com (H.L.R.); Massimo.Bionaz@oregonstate.edu (M.B.)

2 Department of Statistics, College of Science, Oregon State University, Corvallis, OR 97331, USA; Duo.Jiang@oregonstate.edu

3 BIOMIN Research Center, Technopark 1, 3430 Tulln, Austria; barbara.doupovec@dsm.com (B.D.); johannes.faas@dsm.com (J.F.); dian.schatzmayr@dsm.com (D.S.)

4 Department of Clinical Sciences, Carlson College of Veterinary Medicine, Oregon State University, Corvallis, OR 97331, USA; Charles.Estill@oregonstate.edu

5 Department of Environmental \& Molecular Toxicology, College of Agricultural Sciences, Oregon State University, Corvallis, OR 97331, USA

* Correspondence: Jennifer.Duringer@oregonstate.edu; Tel.: +1-541-737-9414

check for

updates

Citation: Roberts, H.L.; Bionaz, M.; Jiang, D.; Doupovec, B.; Faas, J.; Estill, C.T.; Schatzmayr, D.; Duringer, J.M. Effects of Deoxynivalenol and Fumonisins Fed in Combination to Beef Cattle: Immunotoxicity and

Gene Expression. Toxins 2021, 13, 714. https: / / doi.org/10.3390/ toxins13100714

\section{Received: 27 August 2021}

Accepted: 5 October 2021

Published: 10 October 2021

Publisher's Note: MDPI stays neutral with regard to jurisdictional claims in published maps and institutional affiliations.

Copyright: (c) 2021 by the authors. Licensee MDPI, Basel, Switzerland. This article is an open access article distributed under the terms and conditions of the Creative Commons Attribution (CC BY) license (https:/ / creativecommons.org/licenses/by/ $4.0 /)$.

\begin{abstract}
We evaluated the effects of a treatment diet contaminated with $1.7 \mathrm{mg}$ deoxynivalenol and $3.5 \mathrm{mg}$ fumonisins (B1, B2 and B3) per $\mathrm{kg}$ ration on immune status and peripheral blood gene expression profiles in finishing-stage Angus steers. The mycotoxin treatment diet was fed for a period of 21 days followed by a two-week washout period during which time all animals consumed the control diet. Whole-blood leukocyte differentials were performed weekly throughout the experimental and washout period. Comparative profiles of $\mathrm{CD} 4^{+}$and $\mathrm{CD} 8^{+} \mathrm{T}$ cells, along with bactericidal capacity of circulating neutrophils and monocytes were evaluated at $0,7,14,21$ and 35 days. Peripheral blood gene expression was measured at 0,7,21 and 35 days via RNA sequencing. Significant increases in the percentage of $\mathrm{CD}^{-} \mathrm{CD}^{+} \mathrm{T}$ cells were observed in treatment-fed steers after two weeks of treatment and were associated with decreased CD4:CD8 T-cell ratios at this same timepoint $(p \leq 0.10)$. No significant differences were observed as an effect of treatment in terms of bactericidal capacity at any timepoint. Dietary treatments induced major changes in transcripts associated with endocrine, metabolic and infectious diseases; protein digestion and absorption; and environmental information processing (inhibition of signaling and processing), as evaluated by dynamic impact analysis. DAVID analysis also suggested treatment effects on oxygen transport, extra-cellular signaling, cell membrane structure and immune system function. These results indicate that finishing-stage beef cattle are susceptible to the immunotoxic and transcript-inhibitory effects of deoxynivalenol and fumonisins at levels which may be realistically encountered in feedlot situations.
\end{abstract}

Keywords: Fusarium; beef cattle; immune function; RNA-Seq; deoxynivalenol; fumonisin

Key Contribution: In beef cattle exposed to dietary $1.7 \mathrm{mg}$ deoxynivalenol and $3.5 \mathrm{mg}$ fumonisins (per $\mathrm{kg}$ ration), significant shifts in T-helper and cytotoxic T-cell populations indicate that the adaptive immune system in these animals may be altered by Fusarium mycotoxin exposure. Both DIA and DAVID analysis tools showed consistent inhibition of focal adhesion, extra-cellular matrix signaling, and PI3K-Akt signaling pathways. Cell organization (collagen, keratin, fibril) and oxygen and heme transport gene networks were also affected.

\section{Introduction}

Several metabolites of Fusarium fungi, collectively known as Fusarium toxins, have demonstrated deleterious effects in humans and livestock that target immune and hepatic 
systems [1,2]. The Fusarium toxins deoxynivalenol (DON) and fumonisins (FUM) often cooccur in animal feed products [3-5]. As such, research into simultaneous exposures to these compounds has increased, with special attention given to their long-term effects in livestock species that ultimately enter the human food chain. Ruminants, including cattle, are generally considered tolerant of these compounds, yet numerous studies document toxicity in this group of mammals [6-9]. For DON, this can include decreased feed consumption and alterations in the rumen such as decreased NDF digestibility; for FUM, changes in liver enzymes and markers related to metabolism have been noted [10]. A review on mycotoxin effects in ruminants cited a need for research in this group of livestock, particularly studies that consider the simultaneous occurrence of dietary mycotoxins, as they can have additive, antagonistic or synergistic effects [10].

Behind the toxic potential of these compounds are highly conserved molecular mechanisms. As a trichothecene, DON disrupts transcription and cellular repair through processes which have been previously well reviewed [11]. One of these sequelae-inhibition of protein synthesis-appears to target the immune and gastro-hepatic systems $[8,12-15]$. The pathogenesis of dysfunction from fumonisin exposure is linked to the inhibition of serine palmitoyltransferase, which alters sphingolipid metabolism and disrupts cell membranes and function [1,16-18]. In addition to the more direct impact of Fusarium toxins on target organs, the concurrent alterations of the transcriptome observed in organs [19] and cell cultures [20,21], or a delayed release of these toxins following tissue deposition [22,23], may contribute to long-term damage that remains unresolved even after removal of the contaminated feed source.

This work was part of a larger experiment [24], the purpose of which was to both quantify the effects of dietary DON and FUM co-exposure typically seen in regimens for finishing cattle and evaluate the adequacy of a clearance period in ameliorating these effects. To represent a dose relevant to these economically important production animals, the toxin levels chosen were based on previous surveys of commonly used feedstuffs while not exceeding guidance levels [3-5]. In the present study, we utilized a global transcriptomic approach of whole blood to investigate changes in gene expression pathways which may influence long-term animal health through a three-week mycotoxin exposure period, followed by a two-week clearance period. While we examined whole system changes through the Dynamic Impact Approach (DIA) and the Database for Annotation, Visualization and Integrated Discovery (DAVID), we were particularly focused on immune and metabolic pathway alterations. Systemic modulation of immune function was further characterized by flow cytometric and biochemical analysis of peripheral blood throughout the treatment and clearance periods. These data will serve the beef cattle industry's efforts to understand, quantify, and ultimately overcome the risks associated with Fusarium toxins in finishing cattle through the development of targeted management practices.

\section{Results and Discussion}

\subsection{White Blood Cell Population Dynamics and Functionality}

A notable increase in total white blood cells (WBC) was observed in treatmentfed steers beginning at week 3 , which continued throughout the remainder of the trial (Figure 1); however, these changes were not significant $(p>0.10)$. Changes in major leukocyte types (lymphocytes, neutrophils, and monocytes), as a percentage of total leukocyte count, were not significantly affected by diet $(p>0.10$; Figure 1$)$, though numerical patterns reversed for all three major subsets between two and three weeks of treatment. Per the diagnostic laboratory from where these results were obtained, average values for controlfed steers were within a normal range (white blood cell counts 4000-12,000 cells/ $\mu \mathrm{L}$, with lymphocytes $45-75 \%$, monocytes $2-7 \%$ and neutrophils $15-45 \%$ of white blood cells) at all time points, while total white blood cell counts for treatment-fed animals were, on average, elevated past week one of the trial. In comparative in vitro studies of peripheral blood mononuclear cells obtained from cattle, swine and chickens, Novak et al. (2018) concluded that bovine cells were more sensitive to DON than those from other livestock species [13]. 
Our results indicate that $1.7 \mathrm{mg}$ DON and $3.5 \mathrm{mg}$ FUM per kg of total ration were insufficient to cause significant cytotoxic effects on circulating mononuclear leukocytes in these animals.
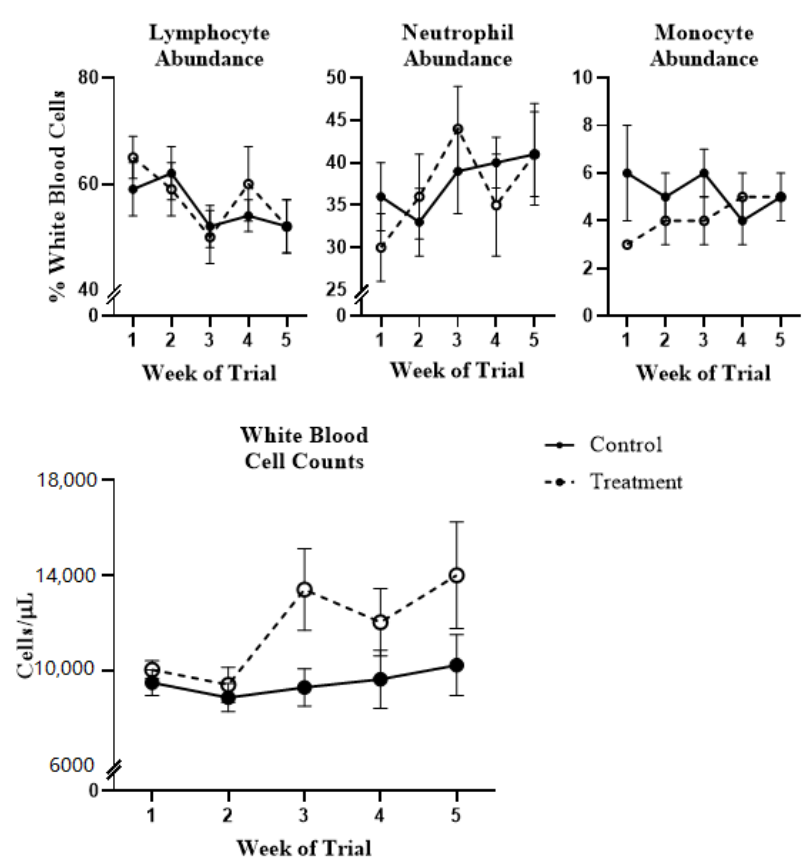

$\rightarrow$ Control

-.. Treatment

Figure 1. White blood cell differential (lymphocyte, neutrophil and monocyte abundance as a percentage of white blood cells, total white blood cells / $\mu \mathrm{L}$ blood) of beef cattle exposed to Fusarium mycotoxins for 21 days, followed by a 14-day clearance period. Values are observed means \pm standard errors with significance from model described in the materials and methods. Reference values provided by diagnostic laboratory for these tests: white blood cell counts $4000-12,000 / \mu \mathrm{L}$, with lymphocytes $45-75 \%$, monocytes $2-7 \%$ and neutrophils $15-45 \%$ of white blood cells.

Neutrophil and monocyte bactericidal functions did not significantly differ between treatment- and control-fed steers throughout the experiment ( $p>0.10$; Figure 2). Interestingly, phagocytic capacity of granulocytes from all steers was substantially reduced by week 5, while monocyte activity began decreasing even earlier in the study (Figure 2).

From the present work, it appears that this may be a natural progression of the fattening of beef steer, which may increase susceptibility to pathogenic bacteria at this critical stage of production. The data presented by Allen et al. (2001) also showed substantially reduced phagocytic activity in finishing-stage steers, regardless of prior mycotoxin exposure. While the effect of body condition or growth status on these outcomes was not formally evaluated in this study, these findings are deserving of future research into the vulnerability of these high-cost, high-demand animals at this state of extreme physiological demand.

In control-fed steers only, $\mathrm{CD} 8^{+}$lymphocytes decreased during the first two weeks of the treatment period (Figure 3), while treatment-fed animals had significantly higher. 

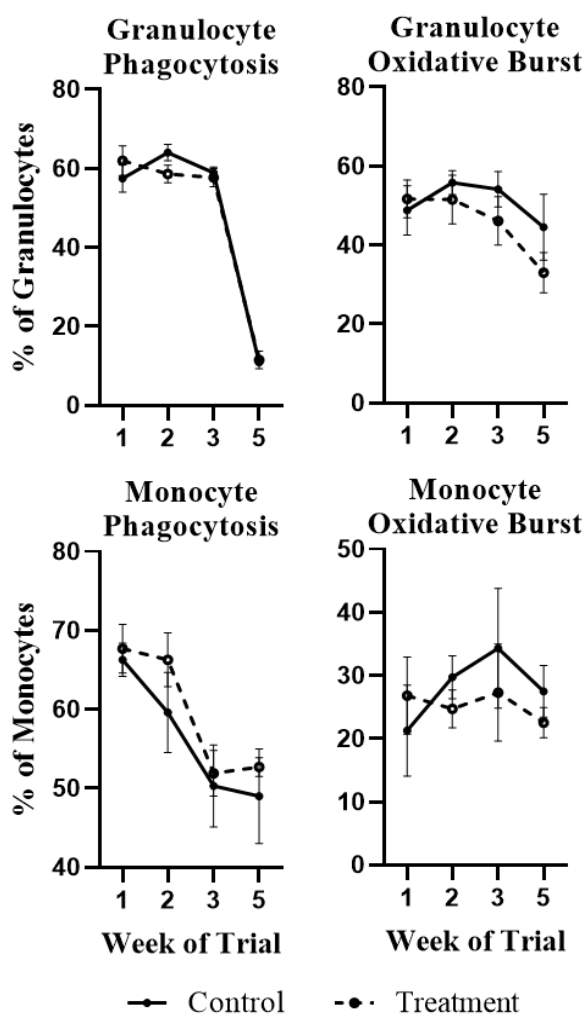

Figure 2. Phagocytosis and oxidative burst in granulocytes and monocytes of beef cattle exposed to Fusarium mycotoxins for 21 days, followed by a 14-day clearance period. Values are observed as means \pm standard errors with significance from model described in the materials and methods.

$\mathrm{CD} 4^{-} \mathrm{CD} 8^{+}$lymphocyte populations during week 2 ( $p \leq 0.10$; Figure 3$)$. This change in the $\mathrm{CD} 8^{+}$lymphocyte population ultimately led to significantly reduced $\mathrm{CD} 4 \mathrm{CD} 8$ ratios in treatment-fed steers during the same time period $(p \leq 0.10$; Figure 3$)$. The decreased $\mathrm{CD}^{+}$population in control-fed animals is assumed to be an adaptive response to the high-starch diet and increased body condition of control-fed animals of the current study. Fusarium toxins such as DON are generally considered pro-inflammatory at low doses or early on in exposure, with anti-inflammatory consequences following prolonged toxicity [11]. Total leukocyte and neutrophil percentages indicate a more grossly sustained pro-inflammatory response, with $\mathrm{CD} 4^{-} \mathrm{CD} 8^{+}$lymphocytes and the $\mathrm{CD} 4: \mathrm{CD} 8$ ratio responding more sensitively. Decreased proliferation of $\mathrm{CD} 4^{-} \mathrm{CD} 8^{+}, \mathrm{CD} 4^{+} \mathrm{CD} 8-$ and $\mathrm{CD} 4^{+} \mathrm{CD} 8^{+}$ porcine T cells with DON exposure at levels greater than $0.4 \mu \mathrm{M}$ and reduced proliferation of peripheral blood mononuclear cells (PBMCs, primarily lymphocytes of unknown CD4/8 phenotypes) in cattle at DON levels as low as $0.21 \mu \mathrm{M}$ have been observed $[13,25]$. While true absorption of DON in steers of this study is unknown, even a conservative estimate of blood volume (425 kg average body weight, $9 \mathrm{~kg}$ TMR intake/day [24]) would conclude that cytotoxic DON levels are reported to perturb PBMCs from this species [13] would be attainable only with a high absorption and distribution of the parent toxin. A previous work by Taranu (2010) reported that FUM was a very poor inhibitor of PBMC proliferation; however, the combined effects of DON and FUM on PBMCs in this species have not been explored. Taken together, these results suggest that the CD4:CD8 ratio could be a candidate biomarker of early DON exposure in beef cattle, which may be attenuated by co-occurring FUM toxicity. These results support previous literature citing the sensitivity of lymphocytes to this class of toxins $[12,13,24]$. Additional validation studies with a larger number of samples across more stratified doses are warranted to further explore this possibility. 

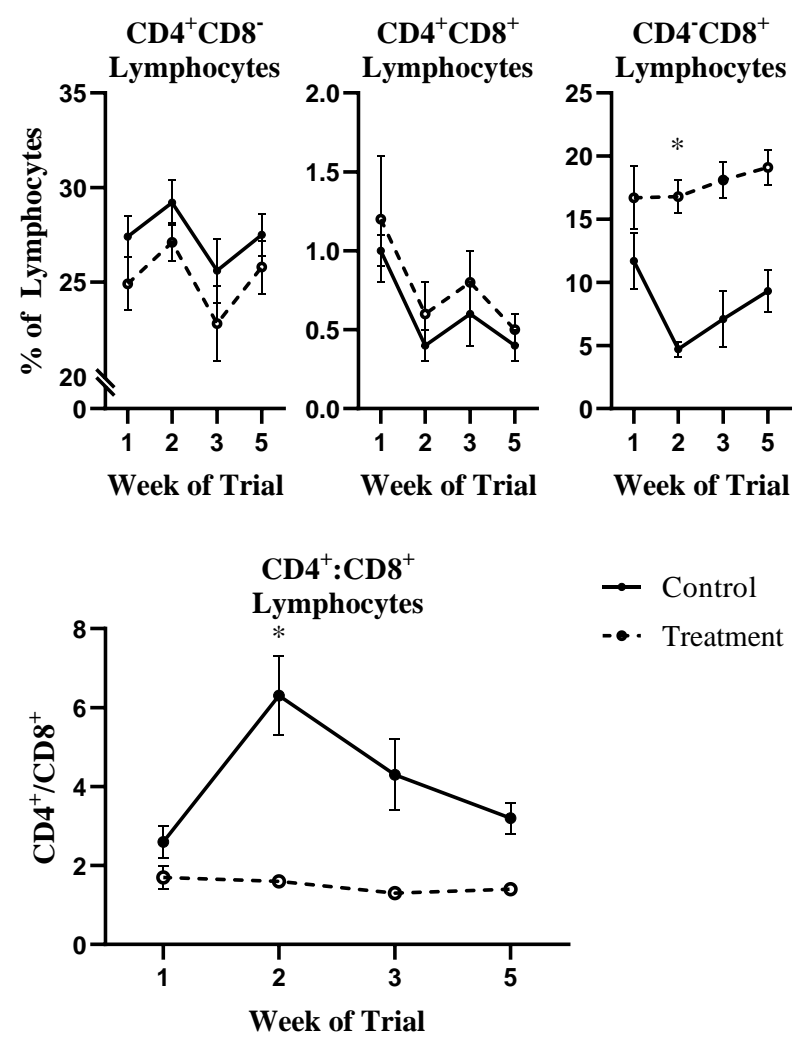

Figure 3. Lymphocyte CD4 and CD8 expression in beef cattle exposed to Fusarium mycotoxins for 21 days, followed by a 14-day clearance period on. Values are observed means \pm standard errors with significance from model described in the materials and methods. ${ }^{*}$ Adjusted $p$-value $\leq 0.10$.

\subsection{RNA-Seq}

Principal components analysis of the RNA-Seq data revealed that the third principal component exhibited a significant increase in the dietary treatment group compared with the control group (Bonferroni-adjusted $p=0.0207$, Supplementary Figure S1). This suggests that there were overall variation patterns in gene expressions caused by the dietary treatment. RNA-Seq analysis revealed 217 differentially expressed genes (DEG; FDR $\leq 0.05$ ) between treatment- and control-fed steers, with mycotoxin exposure broadly leading to down-regulation of annotated features (Supplementary Tables S3 and S4).

\subsection{DIA Results}

According to the DIA summary (Figure 4; Supplementary Materials), the most impacted pathways were "Human Diseases," followed by "Organismal Systems" and "Environmental Information Processing." The high impact of those pathways was due to the significance of a few sub-categories of pathways, which all demonstrated inhibition in the treatment group.

\subsubsection{Human Diseases}

Among the Human Diseases sub-categories, "Endocrine and Metabolic Diseases" and pathways related to "Infectious Diseases" were the most impacted and were inhibited in the treatment group. It is unclear the reason for which the latter pathway was so significantly altered; however, we speculate that this may indicate a reduced immune activation/capacity. For "Endocrine and Metabolic Diseases," the "AGE-RAGE Signaling Pathway in Diabetic Complications" was the most significantly inhibited and was among the most impacted pathways for all time points. This pathway is associated with response to oxidative stress and inflammation [26]; the results demonstrated here indicate an inhibition of cellular response to signaling cues in animals exposed to the DON/FUM diet which persisted throughout the clearance period. 


\begin{tabular}{|c|c|c|c|}
\hline \multirow{2}{*}{$\frac{\text { Category }}{1 . \text { Metabolism }}$} & \multirow[t]{2}{*}{ Day 7} & \multirow[t]{2}{*}{ Day 21} & \multirow[t]{2}{*}{ Day 35} \\
\hline & & & \\
\hline $1.0 \mathrm{Global}$ and overview maps & & & \\
\hline 1.1 Carbohydrate Metabolism & & & \\
\hline 1.2 Energy Metabolism & & & \\
\hline 1.3 Lipid Metabolism & & & \\
\hline 1.4 Nucleotide Metabolism & & & \\
\hline 1.5 Amino Acid Metabolism & & & \\
\hline 1.6 Metabolism of Other Amino Acids & & & \\
\hline 1.7 Glycan Biosynthesis and Metabolism & & & \\
\hline 1.8 Metabolism of Cofactors and Vitamins & & & \\
\hline 1.9 Metabolism of Terpenoids and Polyketides & & & \\
\hline 1.10 Biosynthesis of Other Secondary Metabolites & & & \\
\hline 1.11 Xenobiotics Biodegradation and Metabolism & & & \\
\hline 2. Genetic Information Processing & & & \\
\hline 2.1 Transcription & & & \\
\hline 2.2 Translation & & & \\
\hline 2.3 Folding, Sorting and Degradation & & & \\
\hline 2.4 Replication and Repair & & & \\
\hline 3. Environmental Information Processing & & & \\
\hline 3.1 Membrane transport & & & \\
\hline 3.2 Signal Transduction & & & \\
\hline 3.3 Signaling Molecules and Interaction & & & \\
\hline 4. Cellular Processes & & & \\
\hline 4.1 Transport and Catabolism & & & \\
\hline 4.2 Cell Motility & & & \\
\hline 4.3 Cell Growth and Death & & & \\
\hline 4.4 Cell Communication & & & \\
\hline 4.5 Cell Motility & & & \\
\hline 5. Organismal Systems & & & \\
\hline 5.1 Immune System & & & \\
\hline 5.2 Endocrine System & & & \\
\hline 5.3 Circulatory System & & & \\
\hline 5.4 Digestive System & & & \\
\hline 5.5 Excretory System & & & \\
\hline 5.6 Nervous System & & & \\
\hline 5.7 Sensory System & & & \\
\hline 5.8 Development & & & \\
\hline 5.9 Aging & & & \\
\hline 5.10 Environmental Adaptation & & & \\
\hline 6. Human Diseases & & & \\
\hline 6.1 Cancers: Overview & & & \\
\hline 6.2 Cancers: Specific types & & & \\
\hline 6.3 Immune diseases & & & \\
\hline 6.4 Neurodegenerative Diseases & & & \\
\hline 6.5 Substance dependence & & & \\
\hline 6.6 Cardiovascular Diseases & & & \\
\hline 6.7 Endocrine and metabolic diseases & & & \\
\hline 6.8 Infectious diseases: Bacterial & & & \\
\hline 6.9 Infectious diseases: Viral & & & \\
\hline 6.10 Infectious diseases: Parasitic & & & \\
\hline 6.12 Drug resistance: Antineoplastic & & & \\
\hline Min \% genes in pathway (array vs. genome) Flux $=-50$ & -25 & 0 & \\
\hline
\end{tabular}

Figure 4. DIA results of the summary of Kyoto Encyclopedia of Genes and Genomes (KEGG) pathways affected by differentially expressed genes (DEG). Impact denotes the overall impact of the DEG on the pathways (size of blue bar) and the Flux denotes the overall direction of the impact (i.e., red $=$ activated; dark green $=$ inhibited; orange-light green $=$ moderate change) .

\subsubsection{Organismal System}

The high impact of the Organismal System was clearly due to a strong inhibition of the digestive system. The "Protein Digestion and Absorption" pathway was the most significant influence for this, as it demonstrated strong inhibition. The relevance of this pathway is unclear, since the samples analyzed were circulating blood cells and not tissues associated with the digestive system. The immune system was also highly impacted, showing 
strong inhibition for "Platelet Activation". Platelets are important components for immune activation [27]; thus, inhibition may again indicate an overall trend of immune suppression.

\subsubsection{Environmental Information Processing}

Environmental Information Processing was impacted due to strong inhibition from the "Signaling Molecules and Interaction" pathway. This was the consequence of inhibition of the "Extracellular Matrix (ECM)-Receptor Interaction", while inhibition of "Cellular Growth and Death" was due to inhibition of the "Focal Adhesion" pathway. The P53 pathway was also inhibited, further indicating an alteration of cell cycle control. DON modulation of ECM receptor interactions has been previously noted in both directly exposed cell cultures [28] and, among other tissues, in the thymus of mice exposed to oral DON [29]. Among signaling pathways, the "PI3K-Akt Signaling Pathway" was strongly inhibited, while the "TGF-beta Signaling Pathway" was activated (see Supplementary Tables S24-S26). These alterations may indicate immune depression/suppression. The "PPAR Signaling Pathway," an important pathway in immune differentiation and fate commitment [30], was also inhibited.

\subsubsection{Metabolism}

No key metabolic pathways had clear outcomes related to treatment, with the exception of a small subset of pathways related to the metabolism of amino acids, including 'Thiamine Metabolism' (at both day 7 and 21) and 'Valine, Leucine and Isoleucine Biosynthesis' at day 21. While animals in this study had alterations in performance and digestive and circulating biochemical parameters (which indicated disruption of nutrient utilization [24]), it is difficult to determine if these disruptions were directly linked to the altered metabolism observed in circulating leukocytes. As these data point to a potential intervention in Fusarium toxin pathology, the link between DON/FUM exposure, amino acid metabolism and the systemic immune response warrants further investigation with additional exposure doses and treatment/clearance windows.

\subsection{DAVID Results}

Full DAVID analysis terms and clusters are provided in the Supplementary Materials.

\subsubsection{Oxygen Transport}

Numerous terms associated with heme and oxygen transport were identified from down-regulated genes at day 7 , with this theme persisting throughout the entire treatment and clearance period. While these features were overshadowed by collagen and filamentassociated terms in days 21 and 35, the sustained enrichment of these terms in downregulated genes from treatment-fed steers indicates that iron utilization may be adversely affected by DON/FUM exposure. The disruptive and persistent effects of these toxins on the digestive system have been well reviewed [1,2], and this may be the mechanism by which they altered iron-dependent gene networks in the present study. To our knowledge, more direct changes in oxygen transport have not been previously characterized in this species; however, previous studies have shown alterations to cellular oxygen transport mechanisms by in vitro administration of fumonisins [1,16-18]. Given the rapid growth rate and large musculoskeletal systems of beef steers, the potential for these toxins to systemically alter oxygen transport may increase the risk of cardiovascular and hemolytic events in animals already experiencing the increased physiological burden of feedlot finishing growth state and is deserving of further investigation in animals under additional environmental stressors.

\subsubsection{Extracellular Matrix and Signaling Pathways}

Terms associated with the extracellular space and collagen organization were downregulated in treatment-fed steers, with focal adhesion and the PI3K-Akt signaling pathway being enriched among down-regulated genes at all three timepoints measured. By day 21, 
terms associated with keratin, collagen and intermediate filament were amongst the most highly enriched clusters. Several of these clusters were also present in terms from downregulated genes of treatment-fed steers during the clearance period (day 35). Associated physiological networks have been previously linked to fumonisin toxicity (via disruption of sphingolipid synthesis pathways) [1,16-18].

Of note is the consistent inhibition revealed by both tools (DIA and DAVID) for focal adhesion, ECM signaling, and the PI3K-Akt signaling pathway throughout the study period. Cell adhesion is crucial for optimal functioning of the immune system, especially for extravasation and migration [31]. The PI3K/Akt signaling pathway is pivotal, together with mTOR, for regulating the immune system, as these two proteins are involved in controlling the anti-inflammatory response of immune cells [32]. The role of focal adhesion is less clear; however, we hypothesize that it may be involved in T-cell migration [33].

\subsubsection{Inflammatory Response, Neutrophil Chemotaxis and Immunoglobulin}

Immunotoxic effects of DON/FUM exposure were characterized by the upregulation of inflammatory response terms at days 7 and 21, along with neutrophil chemotaxis terms at day 7. Early toxin exposure was also accompanied by a numerical increase in neutrophil abundance (Figure 1); however, functional assays showed a slight (though insignificant) decrease in granulocyte phagocytosis and oxidative burst (Figure 2). These DEG clusters were not identified at day 35, and it is unclear if the gene expression changes observed here were incapable of resulting in altered performance, or if functional assays did not adequately capture these changes. Immunoglobulin-related pathways were enriched in animals exposed to DON/FUM at day 21. This change did not persist to day 35 and was not observed at day 7. The effects of DON on laboratory animal immunoglobulin levels has been previously reviewed [1,2], and has been shown to result in altered Ig production in a class-dependent manner. Furthermore, previous work has shown that disruptions to antibody production are an important nonlinear toxicological outcome in this species [8,12-15], a conclusion which these data further support.

In general, the data indicate an overall effect on the transcriptome by the dietary mycotoxin treatment in beef cattle. The effect of the treatment on metabolism was negligible. It was interesting to note the consistent inhibition revealed by both tools (DIA and DAVID) for focal adhesion, ECM signaling, and the PI3K-Akt signaling pathway. Cell adhesion is crucial for optimal functioning of the immune system, especially for extravasation and migration [31]. The PI3K/Akt signaling pathway is pivotal, together with mTOR, for regulating the immune system; these two proteins are involved in controlling the anti-inflammatory response of immune cells [32]. The role of focal adhesion is less clear; however, it may be involved with T-cell migration [33].

\section{Conclusions}

This work aimed to quantify transcriptomic and immunological outcomes of multiFusarium toxin exposure in finishing beef cattle at levels similar to those encountered in production scenarios (1.7 $\mathrm{mg}$ DON and $3.5 \mathrm{mg}$ FUM per $\mathrm{kg}$ ration). These results are applicable in both research (immunologists, nutritionists) and clinical (veterinarians, producers) settings, as the commercial value and previously established resistance of this species to DON and FUM indicates that the physiological state (finishing stage or end of production cycle) of these animals may play a role in these outcomes. Deviations in T-helper and cytotoxic cell profiles of treatment-fed steers, along with alterations to several immunomodulatory gene networks, indicate that the immune system of animals in this production class may be altered by Fusarium mycotoxin exposure when compared to control-fed animals of the same genetic background and production stage. Both DIA and DAVID analysis tools showed consistent inhibition of focal adhesion, ECM signaling and PI3K-Akt signaling pathways. Alterations in immune cell chemotaxis, immunoglobulin production and cell membrane structure gene networks were identified as important outcomes of exposure. Unresolved perturbations to cell membrane structure and oxygen and heme transport 
gene networks identified these as potentially chronic toxicological outcomes in this species. These results indicate that exposure to DON and FUM is detrimental to the welfare of finishing steers and may compromise their ability to withstand other stressors such as disease, heat stress or other toxins. In terms of energy utilization and growth, the clearance period used was appropriate to return treatment animals to performance indicators that were similar to control animals; however, a greater clearance period is needed to resolve gene regulation changes observed in beef cattle.

\section{Materials and Methods}

\subsection{Study Design and Diet Formulation}

All animal experiments were approved by the Oregon State University (OSU) Institutional Animal Care and Use Committee (Animal Care and Use Protocol \#4986). In brief, 12 single-sourced Angus beef steers (mean body weight $419 \pm 27 \mathrm{~kg}$ at first sample collection) were communally housed with individual access to assigned rations through a collar-transmitter gate system (American Calan, Northwood, NH, USA). Animals arrived at the research facility 43 days prior to dietary treatments (day -43; Figure 5), at which time each animal was evaluated by a veterinarian and treated metaphylactically with a single dose of Draxxin (Zoetis, Parsipanny, NJ, USA). Feed intake for the week prior to study initiation was used to block all animals to treatment. On day -4 , animals were again evaluated by a veterinarian for overall health. Beginning on day 0 , animals were fed according to their assigned dietary treatment ( $n=6$ animals per treatment).

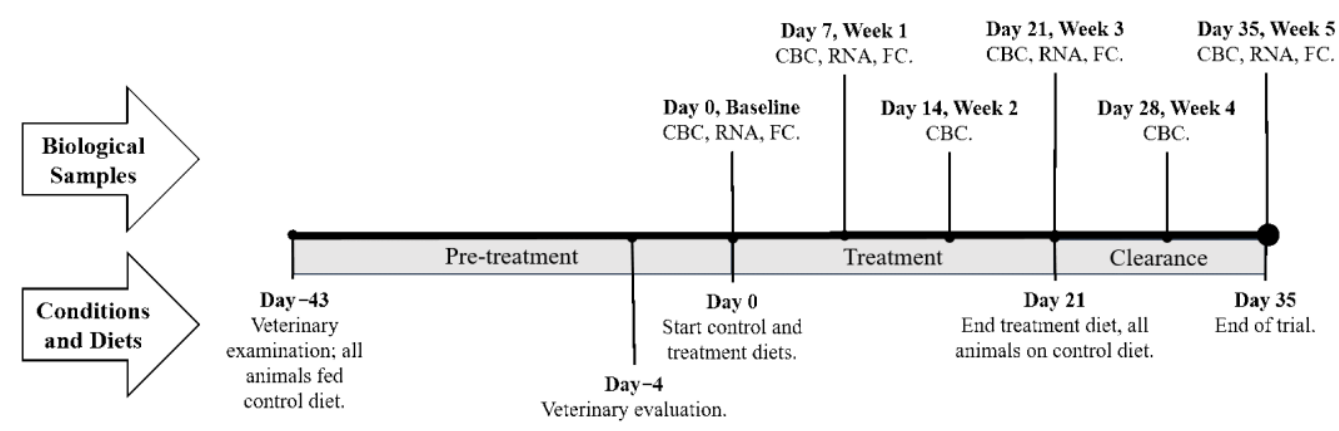

Figure 5. Timeline of experiment used to evaluate the effects of deoxynivalenol and fumonisins fed in combination on beef cattle. $\mathrm{CBC}=$ Blood collection for complete blood count; RNA = Tempus Tube blood collection (for RNA-Seq analysis); $\mathrm{FC}=$ Blood collection for flow cytometric analysis.

Treatments differed in the ground corn constituent of the total mixed ration (TMR), which served as a source of natural toxin contamination, in addition to a fungal culture (produced by BIOMIN Research Center, Tulln, Austria). Average Fusarium toxin concentrations of final rations were $1.7 \pm 0.2 \mathrm{mg}$ for DON and $3.5 \pm 0.3 \mathrm{mg}$ for FUM per $\mathrm{kg}$ TMR for the treatment diet and $0.2 \pm 0.1 \mathrm{mg}$ for DON and $0.2 \pm 0.2 \mathrm{mg}$ for FUM per $\mathrm{kg}$ TMR for the control diet. FUM levels are reported as combined fumonisins B1, B2 and B3. Detailed mycotoxin analysis of TMR can be found in the Supplementary Materials; this did not include examination for modified forms. These doses were selected to mirror those that beef cattle would ingest in a U.S. feedlot, where co-exposure of animals to multi-mycotoxins is common, but below guidance limits [3,5]. Throughout the study, TMRs from both groups were sampled each day, pooled by week, and sent to an accredited commercial laboratory for mycotoxin quantitation as described previously [24]; of the mycotoxins included in the panel, only nivalenol and ZEN were detected above the detection limit in the treatment samples (average 242 and $85 \mathrm{ug} / \mathrm{kg}$ ). Aflatoxins, ochratoxin, acetyl-deoxynivalenol, fusarenon-X, HT-2 toxin, T-2 toxin, neosolaniol and diacetoxyscirpenol were below the limit of detection. Assigned diets were administered over the course of three weeks, after which time all animals were offered the control ration for a clearance period of two weeks (Figure 5). Additional diet formulation details may be found in the Supplementary Materials. Each animal's TMR was provided for ad libitum consumption at approximately the 
same time each morning. Drinking water was also supplied ad libitum. Feed consumption and other production data for these animals may be found in Duringer et al., (2020) [24].

\subsection{Blood Collection}

Blood was collected from each steer at approximately the same time of morning as outlined in Figure 5. Venous blood from a jugular vein was collected into BD Vacutainer ${ }^{\circledR}$ (Becton, Dickinson and Company, Franklin Lakes, NJ, USA) collection tubes containing either EDTA (complete blood counts, CBC) or sodium heparin (flow cytometric analyses) as an anticoagulant and into Tempus ${ }^{\circledR}$ (Thermo-Fisher Scientific, Waltham, MA, USA) blood collection tubes for RNA isolation. Upon blood collection, Vacutainer tubes were inverted gently several times and Tempus tubes were shaken vigorously for 60 seconds before being stored at room temperature for transport. Heparinized blood was completely processed (and cells fixed) within $4 \mathrm{~h}$; EDTA anti-coagulated blood was immediately transported to an accredited veterinary diagnostic laboratory for CBC and Tempus tubes were stored at $-80{ }^{\circ} \mathrm{C}$ for processing at the end of the trial. $\mathrm{CBC}$ s were performed on a Siemens ADVIA 120 Hematology System (Munich, Germany), with the white blood cell differential count performed manually using 100 cells.

\subsection{Reagents and Laboratory Conditions}

For all assays described below, centrifugation was completed at room temperature, unless otherwise noted. Ultrapure water $(18 \mathrm{~m} \Omega)$ was acquired from an ELGA Ultra PureLab water purification system (Cary, NC, USA).

\subsection{RNA Isolation, Sequencing, and Pathway Analysis}

Total RNA was extracted using a Tempus ${ }^{\mathrm{TM}}$ Spin RNA Isolation Kit (Thermo-Fisher Scientific), per the manufacturer's directions. Following isolation, all further RNA work was completed using the facilities at the OSU Center for Genome Research and Biocomputing (Corvallis, OR, USA). An Agilent 2100 Bioanalyzer (Agilent Technologies, Santa Clara, CA, USA) and an RNA Nano Chip (Agilent Technologies) were used to quantify RNA quality and concentration. All samples for cDNA library prep had satisfactory RNA Integrity Numbers of $\geq 8.0$.

Strand-specific cDNA libraries were prepared with poly-A selection for each sample, checked with an Agilent TapeStation 4200 (Agilent Technologies), and validated with single dilution qPCR. Each library was sequenced using an Illumina HiSeq 3000 (Illumina, Inc., San Diego, CA, USA) with a 100 bp read length (single end). Pseudoalignment of each cDNA library to the Bos taurus reference genome (UMD3.1.1) [34] was completed using kallisto v. 0.42.4 [35] and converted to gene-level estimates through the tximport R package [36].

\subsection{Bioinformatics Analyses}

Details of the Dynamic Impact Approach have been previously described [37]. Briefly, the DIA attempts to capture the biological impact of any condition as inferred through the combination of the proportion of DEG compared to all measured transcripts, the significance of the change of the DEG as $-\log _{10}$ of the P-value, and the $\log _{2}$ of the expression ratio to obtain an impact. This can then be interpreted as the overall impact of the condition studied on the genes associated with a particular term; in the present manuscript, only KEGG pathways were used. The DIA also delineates the direction of the impact (or flux), i.e., the overall direction of the impacted term (e.g., pathway) as being either activated or inhibited based on a combination of the expression ratio, significance of the change, and the effect of each protein coded by the affected genes on the specific term (e.g., pathway). For the current study, all annotated and measured genes were uploaded into the DIA using the following criteria: FDR cut-off $=0.05$ and a minimum of four genes in each pathway. DIA functions through Excel (Microsoft, Redmond, WA USA) and can be requested from 
co-author Dr. Bionaz. An online version is available here https:/ / dynamicimpactapproach. shinyapps.io/diarelease1/ (accessed on 15 January 2021).

The enrichment analysis of the DEG was performed using the Database for Annotation, Visualization and Integrated Discovery (DAVID) v.6.8 [38,39]. The whole annotated and measured transcriptome in the experiment was used as background and the default criteria were selected. The analysis was performed for each time point using all DEG (FDR $\leq 0.05$ ), plus an analysis of only the up-regulated DEG and only the down-regulated DEG to be able to further interpret the results. From DAVID, the Chart and the Cluster list of the biological terms enriched with an EASE scope $<0.1$ were downloaded.

\subsection{Phagocytosis and Oxidative Burst}

Modifications to a commercially available kit $\left(\mathrm{pHrodo}^{\mathrm{TM}}\right.$ Green E. coli, Thermo-Fisher Scientific) were employed to simultaneously evaluate phagocytosis capacity and to differentiate populations of phagocytosing cells (see Supplementary Materials for detailed protocols). Oxidative burst activity of immune cells was assessed following activation by Phorbol 12-myristate 13-acetate, again with modifications to a commercially available kit (Neutrophil/Monocyte Respiratory Burst Assay, Cayman Chemical, Ann Arbor, MC, USA). The red blood cell lysis and antibody incubation steps were adopted from the phagocytosis assay described above.

The sample data was acquired on a CytoFLEX S (Beckman Coulter, Brea, California, USA) flow cytometer using CytExpert software v.2.1 (Beckman Coulter), while data transformation, quality control and gating were performed using FlowJo v.10.4.2 (FlowJo LLC, Ashland, Oregon, USA). Daily quality control beads (Beckman Coulter), the FlowClean $\mathrm{R}$ package [40] and manual gating of singlet events (SSC-A/SSC-H) were implemented for flow cytometry quality control. For comparisons of cell populations participating in phagocytosis and oxidative burst, all samples were compared against control samples using the Overton population comparison feature of FlowJo [41]. CD4 ${ }^{+}$and CD8 ${ }^{+}$cells were manually gated from lymphocytes identified as nucleated, singlet, non-granulocyte, low FSC/SSC events (Figure S2), with gate positions set against control samples.

\subsection{Data Handling and Statistical Analysis}

Statistical analysis of the immunotoxicity data was performed using R v.3.6 [42] and a linear regression model for each of the non-baseline time points. Outliers were removed $(1.5 \times \mathrm{IQR})$, and the final model included the baseline values (for repeated measures) and diet as main effects. RNA-Seq gene-level abundance data were first analyzed using principal components analysis to assess the statistical significance of systematic patterns of gene expression variations in association with the dietary treatment. Variance stabilizing transformation was performed prior to PCA to normalize the gene expression counts. A linear mixed effects model was then fitted on each of the top three principal components (PCs), with dietary treatment and time included as explanatory variables. To detect individual DEG, the RNA-Seq data were analyzed using negative binomial models for each of the non-baseline time points, correcting by each animal's day 0 values. Differential expression analysis was performed using quasi-likelihood tests with the limma and edgeR $\mathrm{R}$ packages [43-45]. To adjust for multiple testing, the Benjamini-Hochberg $(\mathrm{BH})$ procedure was performed for treatment period samples (weeks 1-3) separately from clearance period samples (weeks 4-5). As the aim of this research was to differentiate a large number of outcomes, which may be even subtly altered by the toxin doses used, differences were considered significant at an adjusted $p$-value of $\leq 0.10$, which controls the false discovery rate (FDR) at 0.10 . 
Supplementary Materials: The following are available online at https:/ / www.mdpi.com/article/ 10.3390/toxins13100714/s1: Additional materials and methods: 1 . Scatterplot of second (PC2) and third (PC3) principal components of the RNA-Seq gene abundance data used to evaluate the effects of deoxynivalenol and fumonisins fed in combination on beef cattle; Figure S1. Example gating diagram of CD4 and CD8 lymphocyte profiles used to evaluate the effects of deoxynivalenol and fumonisins fed in combination on beef cattle. Data shown from a single animal at day 21 of the trial; Figure S2. Flow cytometric panel used to quantify phagocytotic capacity and leukocyte populations in beef cattle fed deoxynivalenol and fumonisins in combination; Table S1. Flow cytometric panel to quantify oxidative burst capacity in beef cattle fed deoxynivalenol and fumonisins in combination; Table S2. Differentially expressed genes in beef cattle fed deoxynivalenol and fumonisins in combination; Table S3. Genes expressed in beef cattle fed deoxynivalenol and fumonisins in combination; Table S4. DAVID settings for analysis of differentially expressed genes in beef cattle fed deoxynivalenol and fumonisins in combination; Table S5. DAVID gene expression clustering from beef cattle fed deoxynivalenol and fumonisins in combination; Tables S6-S14. DAVID terms from beef cattle fed deoxynivalenol and fumonisins in combination; Tables S15-S23. Sorted DIA terms from beef cattle fed deoxynivalenol and fumonisins in combination; Tables S24-S26. Mycotoxin analysis of total mixed ration used to evaluate the effects of deoxynivalenol and fumonisins fed in combination on beef cattle; Table S27. Dietary analysis of total mixed ration used to evaluate the effects of deoxynivalenol and fumonisins fed in combination on beef cattle; Table S28. Dietary analysis of total mixed ration used to evaluate the effects of deoxynivalenol and fumonisins fed in combination on beef cattle.

Author Contributions: Conceptualization, J.M.D., H.L.R. and B.D.; methodology, J.M.D., H.L.R., M.B., D.J. and C.T.E.; formal analysis, H.L.R., D.J. and M.B; investigation, J.M.D., H.L.R. and C.T.E.; resources, J.M.D.; data curation, J.M.D. and H.L.R.; writing-original draft preparation, H.L.R.; writing—review and editing, J.M.D., H.L.R., M.B., C.T.E., J.F., D.J., B.D. and D.S.; visualization, J.M.D., H.L.R., M.B. and D.J.; supervision, J.M.D.; project administration, J.M.D. and J.F.; funding acquisition, J.M.D., B.D. and D.S. All authors have read and agreed to the published version of the manuscript.

Funding: This research was funded by BIOMIN Holding GmbH (Getzersdorf, Austria) along with the National Needs Graduate Fellowships, USDA-NIFA grant \#2014-38420-21800.

Institutional Review Board Statement: The study was conducted according to the guidelines of the Declaration of Helsinki and approved by the Institutional Review Board (or Ethics Committee) of Oregon State University (Animal Care and Use Protocol \#4968, approved 4 December 2017).

Acknowledgments: We would also like to acknowledge Seth Spencer and the Farm Services staff of the OSU Department of Animal \& Rangeland Sciences for the care and feeding of the cattle; Linda Blythe, Katherine Swanson and Kari Carola for their assistance with sampling; Gita Cherian for the use of her laboratory space; and Allison Ehrlich for assistance with flow cytometry troubleshooting.

Conflicts of Interest: The authors declare no conflict of interest. B.D., J.F. and D.S. are employed by BIOMIN Holding GmbH (now part of DSM), which operates the BIOMIN Research Center and is a producer of animal feed additives. This had no role in the design of the study; in the collection, analyses or interpretation of data; in the writing of the manuscript; or in the decision to publish the results.

\section{References}

1. Bondy, G.S.; Pestka, J.J. Immunomodulation by Fungal Toxins. J. Toxicol. Environ. Health B Crit. Rev. 2000, 3, 109-143. [CrossRef] [PubMed]

2. Trenholm, H.; Hamilton, R.; Friend, D.; Thompson, B.; Hartin, K. Feeding Trials with Vomitoxin (Deoxynivalenol)-Contaminated Wheat-Effects on Swine, Poultry, and Dairy-Cattle. J. Am. Vet. Med. Assoc. 1984, 185, 527-531.

3. Gruber-Dorninger, C.; Jenkins, T.; Schatzmayr, G. Global Mycotoxin Occurrence in Feed: A Ten-Year Survey. Toxins 2019, 11, 375. [CrossRef] [PubMed]

4. Streit, E.; Naehrer, K.; Rodrigues, I.; Schatzmayr, G. Mycotoxin Occurrence in Feed and Feed Raw Materials Worldwide: Long-Term Analysis with Special Focus on Europe and Asia. J. Sci. Food Agric. 2013, 93, 2892-2899. [CrossRef] [PubMed]

5. U.S. Grains Council. Corn Harvest Quality Report 2016-17. 2016. Available online: https://grains.org/corn_report/corn-harvestquality-report-2015-2016-2/ (accessed on 15 January 2021).

6. Baker, D.C.; Rottinghaus, G.E. Chronic Experimental Fumonisin Intoxication of Calves. J. Vet. Diagn. Invest. 1999, 11, $289-292$. [CrossRef] [PubMed] 
7. Dänicke, S.; Matthäus, K.; Lebzien, P.; Valenta, H.; Stemme, K.; Ueberschär, K.-H.; Razzazi-Fazeli, E.; Böhm, J.; Flachowsky, G. Effects of Fusarium Toxin-Contaminated Wheat Grain on Nutrient Turnover, Microbial Protein Synthesis and Metabolism of Deoxynivalenol and Zearalenone in the Rumen of Dairy Cows. J. Anim. Physiol. Anim. Nutr. 2005, 89, 303-315. [CrossRef] [PubMed]

8. Dänicke, S.; Winkler, J.; Meyer, U.; Kersten, S.; Wernike, K.; Beer, M.; Frahm, J. Antibody Response of Growing German Holstein Bulls to a Vaccination against Bovine Viral Diarrhea Virus (BVDV) Is Influenced by Fusarium Toxin Exposure in a Non-Linear Fashion. Mycotoxin Res. 2018, 34, 123-139. [CrossRef] [PubMed]

9. Korosteleva, S.N.; Smith, T.K.; Boermans, H.J. Effects of Feedborne Fusarium Mycotoxins on the Performance, Metabolism, and Immunity of Dairy Cows. J. Dairy Sci. 2007, 90, 3867-3873. [CrossRef]

10. Gallo, A.; Giuberti, G.; Frisvad, J.C.; Bertuzzi, T.; Nielsen, K.F. Review on Mycotoxin Issues in Ruminants: Occurrence in Forages, Effects of Mycotoxin Ingestion on Health Status and Animal Performance and Practical Strategies to Counteract Their Negative Effects. Toxins 2015, 7, 3057-3111. [CrossRef]

11. Pestka, J.J. Mechanisms of Deoxynivalenol-Induced Gene Expression and Apoptosis. Food Addit. Contam. Part Chem. Anal. Control Expo. Risk Assess. 2008, 25, 1128-1140. [CrossRef]

12. Awad, W.A.; Ruhnau, D.; Hess, C.; Doupovec, B.; Schatzmayr, D.; Hess, M. Feeding of Deoxynivalenol Increases the Intestinal Paracellular Permeability of Broiler Chickens. Arch. Toxicol. 2019, 93, 2057-2064. [CrossRef]

13. Novak, B.; Vatzia, E.; Springler, A.; Pierron, A.; Gerner, W.; Reisinger, N.; Hessenberger, S.; Schatzmayr, G.; Mayer, E. Bovine Peripheral Blood Mononuclear Cells Are More Sensitive to Deoxynivalenol than Those Derived from Poultry and Swine. Toxins 2018, 10, 152. [CrossRef]

14. Taranu, I.; Marina, D.E.; Burlacu, R.; Pinton, P.; Damian, V.; Oswald, I.P. Comparative Aspects of in Vitro Proliferation of Human and Porcine Lymphocytes Exposed to Mycotoxins. Arch. Anim. Nutr. 2010, 64, 383-393. [CrossRef]

15. Xu, L.; Eicher, S.D.; Applegate, T.J. Effects of Increasing Dietary Concentrations of Corn Naturally Contaminated with Deoxynivalenol on Broiler and Turkey Poult Performance and Response to Lipopolysaccharide. Poult. Sci. 2011, 90, 2766-2774. [CrossRef]

16. Osweiler, G.D.; Kehrli, M.E.; Stabel, J.R.; Thurston, J.R.; Ross, P.F.; Wilson, T.M. Effects of Fumonisin-Contaminated Corn Screenings on Growth and Health of Feeder Calves. J. Anim. Sci. 1993, 71, 459-466. [CrossRef] [PubMed]

17. Wang, E.; Norred, W.P.; Bacon, C.W.; Riley, R.T.; Merrill, A.H. Inhibition of Sphingolipid Biosynthesis by Fumonisins. Implications for Diseases Associated with Fusarium Moniliforme. J. Biol. Chem. 1991, 266, 14486-14490. [CrossRef]

18. Yin, J.J.; Smith, M.J.; Eppley, R.M.; Page, S.W.; Sphon, J.A. Effects of Fumonisin B1 on Oxygen Transport in Membranes. Biochem. Biophys. Res. Commun. 1996, 225, 250-255. [CrossRef] [PubMed]

19. Reddy, K.E.; Jeong, J.Y.; Lee, Y.; Lee, H.-J.; Kim, M.S.; Kim, D.-W.; Jung, H.J.; Choe, C.; Oh, Y.K.; Lee, S.D. Deoxynivalenol- and Zearalenone-Contaminated Feeds Alter Gene Expression Profiles in the Livers of Piglets. Asian-Australas. J. Anim. Sci. 2018, 31, 595-606. [CrossRef] [PubMed]

20. Fernández-Blanco, C.; Frizzell, C.; Shannon, M.; Ruiz, M.-J.; Connolly, L. An in Vitro Investigation on the Cytotoxic and Nuclear Receptor Transcriptional Activity of the Mycotoxins Fumonisin B1 and Beauvericin. Toxicol. Lett. 2016, 257, 1-10. [CrossRef]

21. He, K.; Zhou, H.-R.; Pestka, J.J. Targets and Intracellular Signaling Mechanisms for Deoxynivalenol-Induced Ribosomal RNA Cleavage. Toxicol. Sci. Off. J. Soc. Toxicol. 2012, 127, 382-390. [CrossRef]

22. Pestka, J.J.; Islam, Z.; Amuzie, C.J. Immunochemical Assessment of Deoxynivalenol Tissue Distribution Following Oral Exposure in the Mouse. Toxicol. Lett. 2008, 178, 83-87. [CrossRef] [PubMed]

23. Prelusky, D.B.; Trenholm, H.L.; Savard, M.E. Pharmacokinetic Fate of 14C-Labelled Fumonisin B1 in Swine. Nat. Toxins 1994, 2, 73-80. [CrossRef] [PubMed]

24. Duringer, J.M.; Roberts, H.1.; Doupovec, B.; Faas, J.; Estill, C.T.; Jiang, D.; Schatzmayr, D. Effects of Deoxynivalenol and Fumonisins Fed in Combination on Beef Cattle: Health and Performance Indices. World Mycotoxin J. 2020, 13, 533-543. [CrossRef]

25. Vatzia, E.; Pierron, A.; Hoog, A.M.; Saalmüller, A.; Mayer, E.; Gerner, W. Deoxynivalenol Has the Capacity to Increase Transcription Factor Expression and Cytokine Production in Porcine T Cells. Front. Immunol. 2020, 11, 2009. [CrossRef]

26. Kierdorf, K.; Fritz, G. RAGE Regulation and Signaling in Inflammation and Beyond. J. Leukoc. Biol. 2013, 94, 55-68. [CrossRef]

27. Cox, D.; Kerrigan, S.W.; Watson, S.P. Platelets and the Innate Immune System: Mechanisms of Bacterial-Induced Platelet Activation. J. Thromb. Haemost. JTH 2011, 9, 1097-1107. [CrossRef]

28. Del Favero, G.; Woelflingseder, L.; Janker, L.; Neuditschko, B.; Seriani, S.; Gallina, P.; Sbaizero, O.; Gerner, C.; Marko, D. Deoxynivalenol Induces Structural Alterations in Epidermoid Carcinoma Cells A431 and Impairs the Response to Biomechanical Stimulation. Sci. Rep. 2018, 8, 11351. [CrossRef]

29. van Kol, S.W.M.; Hendriksen, P.J.M.; van Loveren, H.; Peijnenburg, A. The Effects of Deoxynivalenol on Gene Expression in the Murine Thymus. Toxicol. Appl. Pharmacol. 2011, 250, 299-311. [CrossRef]

30. Christofides, A.; Konstantinidou, E.; Jani, C.; Boussiotis, V.A. The Role of Peroxisome Proliferator-Activated Receptors (PPAR) in Immune Responses. Metabolism 2021, 114, 154338. [CrossRef]

31. Harjunpää, H.; Llort Asens, M.; Guenther, C.; Fagerholm, S.C. Cell Adhesion Molecules and Their Roles and Regulation in the Immune and Tumor Microenvironment. Front. Immunol. 2019, 10, 1078. [CrossRef]

32. Weichhart, T.; Säemann, M.D. The PI3K/Akt/MTOR Pathway in Innate Immune Cells: Emerging Therapeutic Applications. Ann. Rheum. Dis. 2008, 67, iii70-iii74. [CrossRef] 
33. Rose, D.M.; Liu, S.; Woodside, D.G.; Han, J.; Schlaepfer, D.D.; Ginsberg, M.H. Paxillin Binding to the A4 Integrin Subunit Stimulates LFA-1 (Integrin AL 32 )-Dependent T Cell Migration by Augmenting the Activation of Focal Adhesion Kinase/ProlineRich Tyrosine Kinase-2. J. Immunol. 2003, 170, 5912-5918. [CrossRef]

34. Zimin, A.V.; Delcher, A.L.; Florea, L.; Kelley, D.R.; Schatz, M.C.; Puiu, D.; Hanrahan, F.; Pertea, G.; Van Tassell, C.P.; Sonstegard, T.S.; et al. A Whole-Genome Assembly of the Domestic Cow, Bos Taurus. Genome Biol. 2009, 10, R42. [CrossRef]

35. Bray, N.L.; Pimentel, H.; Melsted, P.; Pachter, L. Near-Optimal Probabilistic RNA-Seq Quantification. Nat. Biotechnol. 2016, 34, 525-527. [CrossRef]

36. Soneson, C.; Love, M.I.; Robinson, M.D. Differential Analyses for RNA-Seq: Transcript-Level Estimates Improve Gene-Level Inferences. F1000Research 2015, 4, 1521. [CrossRef] [PubMed]

37. Bionaz, M.; Periasamy, K.; Rodriguez-Zas, S.L.; Hurley, W.L.; Loor, J.J. A Novel Dynamic Impact Approach (DIA) for Functional Analysis of Time-Course Omics Studies: Validation Using the Bovine Mammary Transcriptome. PLoS ONE 2012, 7, e32455. [CrossRef]

38. Huang, D.W.; Sherman, B.T.; Lempicki, R.A. Bioinformatics Enrichment Tools: Paths toward the Comprehensive Functional Analysis of Large Gene Lists. Nucleic Acids Res. 2009, 37, 1-13. [CrossRef] [PubMed]

39. Huang, D.W.; Sherman, B.T.; Lempicki, R.A. Systematic and Integrative Analysis of Large Gene Lists Using DAVID Bioinformatics Resources. Nat. Protoc. 2009, 4, 44-57. [CrossRef]

40. Fletez-Brant, K.; Špidlen, J.; Brinkman, R.R.; Roederer, M.; Chattopadhyay, P.K. FlowClean: Automated Identification and Removal of Fluorescence Anomalies in Flow Cytometry Data. Cytom. Part J. Int. Soc. Anal. Cytol. 2016, 89, 461-471. [CrossRef] [PubMed]

41. Overton, W.R. Modified Histogram Subtraction Technique for Analysis of Flow Cytometry Data. Cytometry 1988, 9, 619-626. [CrossRef]

42. R Core Team. R: A Language and Environment for Statistical Computing; R Foundation for Statistical Computing: Vienna, Austria, 2019.

43. McCarthy, D.J.; Chen, Y.; Smyth, G.K. Differential Expression Analysis of Multifactor RNA-Seq Experiments with Respect to Biological Variation. Nucleic Acids Res. 2012, 40, 4288-4297. [CrossRef]

44. Ritchie, M.E.; Phipson, B.; Wu, D.; Hu, Y.; Law, C.W.; Shi, W.; Smyth, G.K. Limma Powers Differential Expression Analyses for RNA-Sequencing and Microarray Studies. Nucleic Acids Res. 2015, 43, e47. [CrossRef] [PubMed]

45. Robinson, M.D.; McCarthy, D.J.; Smyth, G.K. EdgeR: A Bioconductor Package for Differential Expression Analysis of Digital Gene Expression Data. Bioinformatics 2010, 26, 139-140. [CrossRef] [PubMed] 